\title{
RESEARCH AND DEVELOPMENT OF THE INFORMATION MANAGEMENT SYSTEM OF AGRICULTURAL SCIENCE AND TECHNOLOGY TO FARMER BASED ON GIS
}

\author{
Hao Zhang ${ }^{1}$, Lei Xi ${ }^{1}$, Xinming Ma ${ }^{1,2, *}$, Zhongmin $\mathrm{Lu}^{3}$, Yali $\mathrm{Ji}^{1}$, Yanna Ren ${ }^{1}$ \\ 1 College of Information and Management Science, Henan Agricultural University, \\ Zhengzhou, Henan, He Nan, China, 450002 \\ 2 College of Agriculture, Henan Agricultural University, Zhengzhou, Henan, China, 450002 \\ 3 Hua county Center of Agricultural Technology Popularization, Henan, China, 456400 \\ * Corresponding author, Address: College of Information and Management Science, Henan \\ Agricultural University, 63 Agricultural Road, Zhengzhou, 450002, P. R. China, Tel: +86-371- \\ 63558388, Fax: +86-371-63558090, Email: xinmingma@371.net
}

Abstract: With the rapid progress of information technology, more and more people utilize high technology to boost the fast development of the national economy. Since the project of agricultural science and technology to farmer was put into practice by Ministry of Agriculture in 2005, 212 representative counties have been set, 200,000 typical households have been added, and 4 million peasants have enhanced production and increased income. According to the criterion of Software Engineering, the article collected the information of Agricultural science and technology to farmer and the geography information of all villages and towns in HUA county, designed the system structure of Agricultural science and technology to farmer, implemented the information query and management, and realized the special topic analysis to the information distribution by tools of OOP, GIS components and network database, integrating GIS and MIS smoothly. The system has been applied in HUA county, and facilitated information management, analysis and decision-making to the agricultural science and technology to farmer.

Keywords: agricultural science and technology to farmer, GIS, C/S, smooth integration

Zhang, H., Xi, L., Ma, X., Lu, Z., Ji, Y. and Ren, Y., 2008, in IFIP International Federation for Information Processing, Volume 258; Computer and Computing Technologies in Agriculture, Vol. 1; Daoliang Li; (Boston: Springer), pp. 141-150. 


\section{INTRODUCTION}

With the rapid progress of information technology, more and more people utilize high technology to boost the fast development of the national economy (Tang Wan-min, 2006; Yin Li-hui, 2006). Since the project of agricultural science and technology to farmer was put into practice by Ministry of Agriculture in 2005, 212 representative counties have been set, 200,000 typical households have been added, and 4 million peasants have enhanced production and increased income (http://www.gov.cn/jrzg/200610/17/content_415658.htm, 2006). But the Information Management System of agricultural science and technology to farmer has not been developed adequately yet in each of domestic provinces, lacking effective management and analysis. Under this background, the topic of scientific management and analysis to the information of science and technology to farmer is put forward. And 3S(RS, GPS and GIS), which has great predominance of spatial management and analysis, has been widely applied in all kinds of domains, such as transportation, military affairs, agriculture, forestry and so on (Alexander Köninger, 1998; Yan Tai-lai, 2005; Wei Fu-quan, 2004; Li Li-wei, 2006; Gong Jian-ya, 2004). In view of these factors, the item closely followed the project of the science and technology to farmer put into practice by Ministry of Agriculture, researched, designed and developed the information management system of agricultural science and technology to farmer using GIS technology and computer technology.

\section{SYSTEM DESIGN}

Using the virtues of friendly interface, strong simulation, rapid inquiry localization, good expansibility and special spatial analysis of GIS (Wu Lun, 2001; Gong Jian-ya, 2004; Chen Zheng-jiang, 2005) and according to the criterion of software engineering, the article collected the information of Agricultural science and technology to farmer and the geography information of all villages and towns in HUA county, designed the system structure of Agricultural science and technology to farmer, and implemented the query and management of the information of agricultural science and technology to farmer and the special topic analysis of the information distribution by the tools of OOP, the components of GIS and network database, integrating GIS and MIS smoothly. 


\subsection{System Structure}

The system introduced Client/Server/DBMS which separates the logic service from the user connection (Zheng Ke-feng, 2005). Fig. 1 shows the structure of the system. Controlled by unification database interface that is the foundation platform of database, all kinds of spatial data and attribute data are stored by using ArcSDE \& MS SQL Server, which are programmed by using VB and MapObjects.

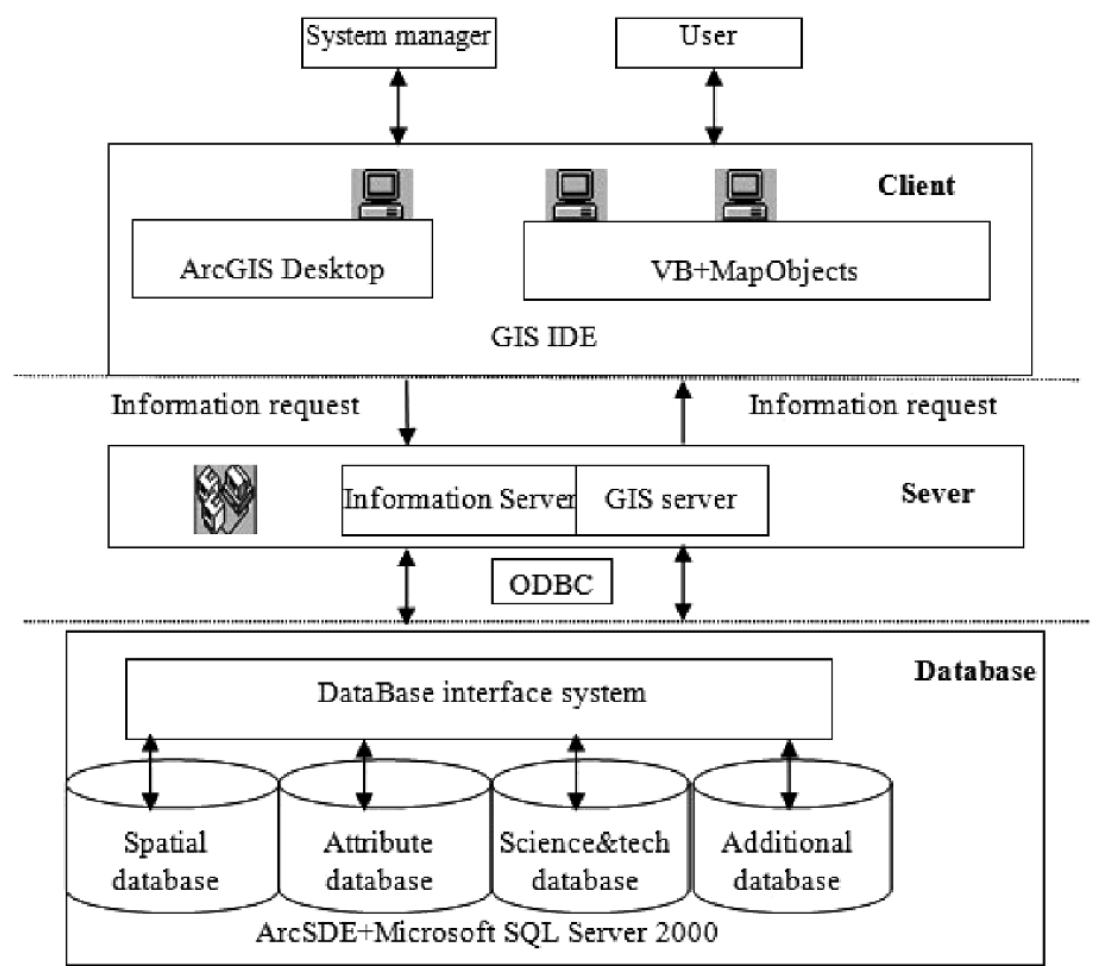

Fig. 1. The structure of the system

Client end is responsible for display of the data and communication with user, which requests information from the server in order to implement all kinds of function, such as information browsing, inquiry, adding, deletion, update and so on. Server end mainly realizes data sharing and data transmission to client. The database platform uses GeoSpatial Database and SQL Server 2000, whose duty is to accept server request operation to the database and transmit data. The $\mathrm{C} / \mathrm{S}$ pattern needs to install server software in the server end and client software in the client end. The server data such as spatial data, the attribute data, the information of agricultural science and 
technology to farmer and other information are saved in the server end, the client data are put on the Client end. When client end needs to request data of server end, it will send out the request through the local network to server, server end confirms connection, accepts and processes the request information, and returns the processed result to client end.

\subsection{System Function}

The main function of the information management system of agricultural science and technology to farmer consists of the information inquiry, the analysis to the special topic, the attribute database management, the spatial database management, and system maintenance and help. Fig. 2 shows the system function structure.

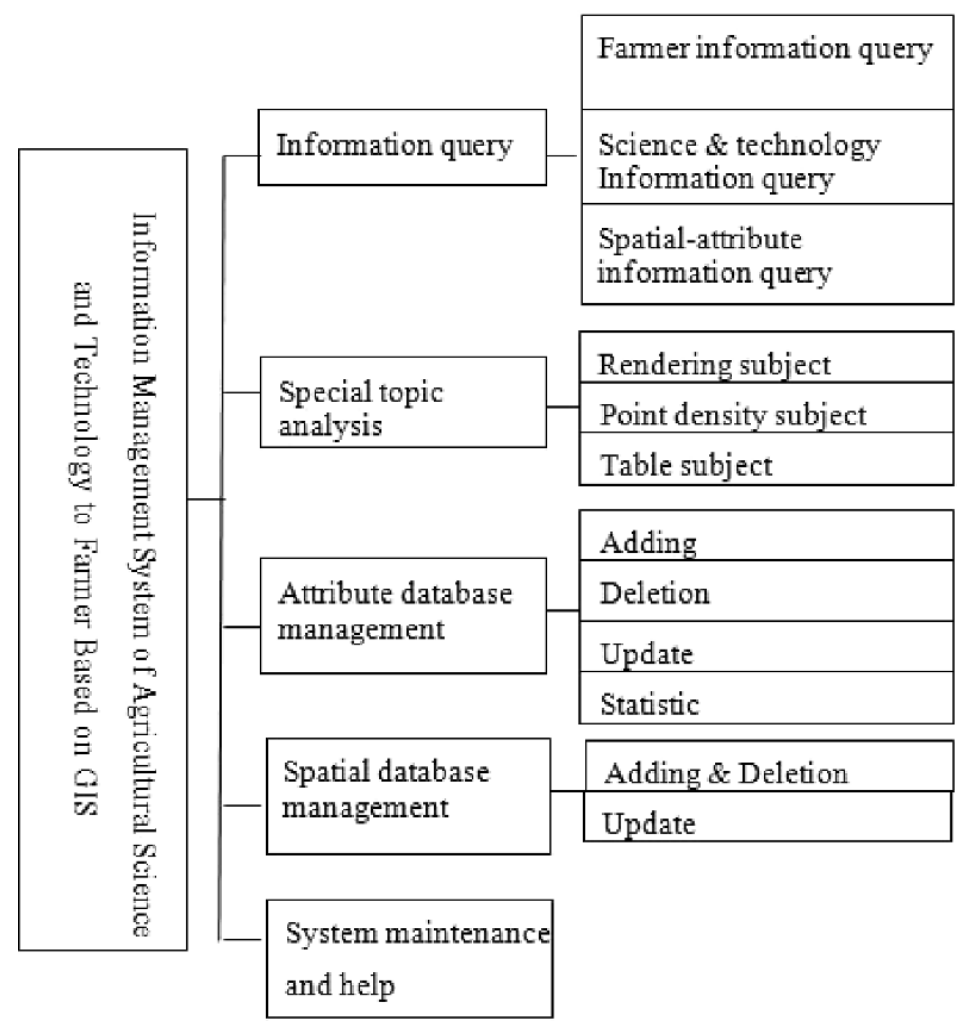

Fig. 2. The function of the system

(1) Information inquiry. It includes farmer information inquiry, the information inquiry of science and technology to farmer, the spatial and attribute information inquiry and so on. 
(2) Special topic analysis. It makes dynamic color topic, point density topic, table topic and so on, and carrying on kinds of statistic, analysis and the decision-making according to the special data chosen by user.

(3) Attribute database management. It includes adding, deletion and update to attribute data.

(4) Spatial database management. It includes adding, deletion and update to spatial data.

(5) System maintenance and help. It includes system information maintenance, system operation manual and so on.

\section{SYSTEM REALIZATION}

System realization includes database design and the application of key technology.

\subsection{Database Design}

Database design is the key of effective working and function implement of GIS and MIS (Yang Bao-zhu, 2005). This system database designed by adopting E-R model mainly includes the spatial character database, the attribute characteristic database, the database of agricultural science and technology to farmer, the peasant information database, the system maintenance information database and so on. Fig. 3 shows the database structure of agricultural science and technology to farmer. Fig. 4 shows the

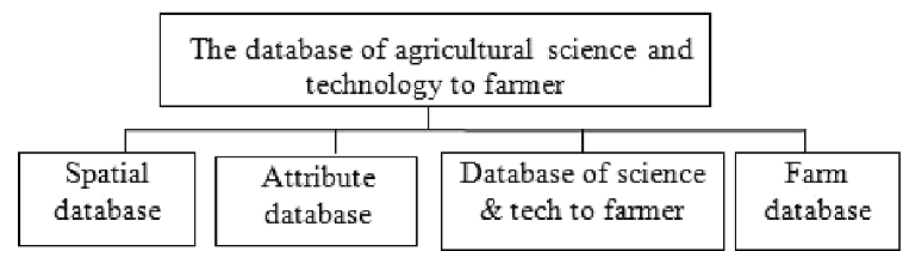

Fig. 3. The database structure of agricultural science and Technology to Farmer

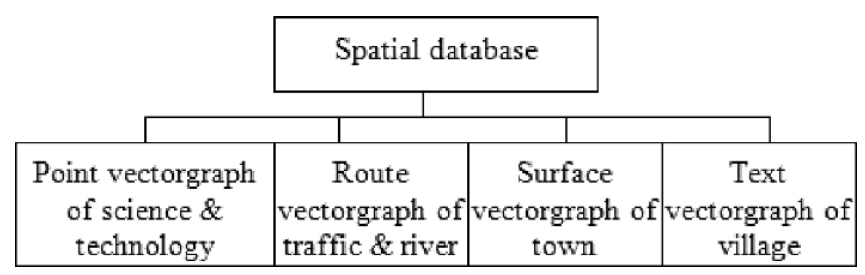

Fig. 4. The database structure of spatial character 


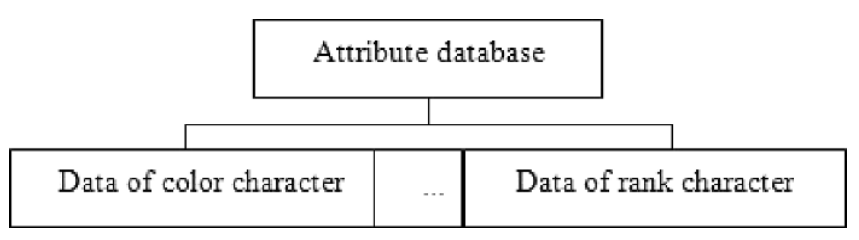

Fig. 5. The database structure of attribute character

database structure of spatial character, and Fig. 5 shows the database structure of attribute character.

The content classification and the structural design of Attribute database and the information database of agricultural science and technology to farmer are the successful and unsuccessful key factor of system development.

\subsubsection{Spatial Database}

Spatial database is created by using 1:10,000-scale electronic map. The content of spatial database mainly includes: the point vector map of the information distribution of science and technology to farmer, the line vector map of transportation and rivers, the surface vector map of villages and towns, the village text vector map and so on. The information distribution of science and technology to farmer are collected according to the unit of village and town.

\subsubsection{Attribute Database}

Attribute database of each kind of vector map of HUA county mainly comprises the information of geography object which has nothing to do with the spatial position, such as time, color, quality, rank, type and so on.

\subsubsection{Database of Agricultural Science and Technology to Farmer}

The content of the database of agricultural science and technology to farmer includes: serial_number, name, sex, birthday, culture_level, population _quantity, farmer_quantity, representative_crops_type, plant_scale, cultivation _scale, Cultivation_quomodo, cropping_quomodo, weeding_quomodo, plant_ di_sease_therapeusis, average_income_per_year, telepho_ne, Internet_or_not, village, town, county, province and so on. The database has collected 10,000 farmers' information in 10 counties, 100 towns, and 1,000 villages. Table 1Table 5 shows all fields and six records of the database of agricultural science and technology to farmer (Taking Hua county as an example). 
Table 1. The information of Agricultural Science and Technology to Farmer

\begin{tabular}{llll}
\hline serial_number & name & sex & birthday \\
\hline 11 & Hongsheng Lv & man & Jul-54 \\
12 & Yuejin Lv & man & May-56 \\
13 & Qunli Lv & man & Sep-63 \\
14 & Xianbing Sun & man & May-70 \\
15 & Guobao Lv & man & Mar-63 \\
16 & Junqiang Lv & man & Jul-63 \\
\hline
\end{tabular}

Table 2. The information of Agricultural Science and Technology to Farmer

\begin{tabular}{llll}
\hline culture_level & population & farmer_quantity & representative_crop \\
\hline junior & 10 & 7 & wheat \\
senior & 6 & 4 & wheat \\
junior & 5 & 4 & wheat \\
junior & 3 & 2 & wheat \\
senior & 5 & 2 & wheat \\
junior & 5 & 4 & wheat \\
\hline
\end{tabular}

Table 3. The information of Agricultural Science and Technology to Farmer

\begin{tabular}{llll}
\hline plant_scale & cultivation_scale & Cultivation_quomod & cropping_quomodo \\
\hline 22 & 15 & machine & machine \\
11 & 10 & half-machine & half-machine \\
10 & 8 & half-machine & half-machine \\
6 & 6 & handwork & handwork \\
7 & 6 & half-machine & half-machine \\
15 & 9 & half-machine & half-machine \\
\hline
\end{tabular}

Table 4. The information of Agricultural Science and Technology to Farmer

\begin{tabular}{llll}
\hline average_income & weeding_quomodo & disease_therapeusis & telephone \\
\hline 1950 & herbicide & pesticide & $0372-8425009$ \\
2450 & herbicide & pesticide & $0372-8425016$ \\
2100 & herbicide & pesticide & $0372-8425013$ \\
2250 & handwork & pesticide & $0372-8425159$ \\
2870 & herbicide & pesticide & $0372-8425153$ \\
2150 & herbicide & pesticide & $0372-8425262$ \\
\hline
\end{tabular}


Table 5. The information of Agricultural Science and Technology to Farmer

\begin{tabular}{llll}
\hline Internet_or_not & village & town & county \\
\hline not & Xi_yuan & Ba_li_ying & Hua Xian \\
not & Xi_yuan & Ba_li_ying & Hua Xian \\
not & Xi_yuan & Ba_li_ying & Hua Xian \\
not & Xi_yuan & Ba_li_ying & Hua Xian \\
not & Xi_yuan & Ba_li_ying & Hua Xian \\
not & Xi_yuan & Ba_li_ying & Hua Xian \\
\hline
\end{tabular}

\subsubsection{Additional Database}

Additional database includes farmer information database and system maintenance database and so on. The farmer information database saves and manages the basic farmer information. The basic farmer information content includes: serial number, name, sex, birth, ID_card, culture_level, political_faction, married_or_not, spouse_name, native_place, address, zip_code, telephone, village, town, county, province and so on. The system maintenance database mainly includes: parameter_initialization and user_information, diary_information and so on.

\subsection{Key Technology}

Key technology includes OOP (Object Oriented Programming), GIS components, net database operation and so on.

\subsubsection{Redeveloping By VB and MapObjects}

By MapObjects component loaded in Visual Basic and a series of operations to MapObjects, the system realized map cruise, zoom in, zoom out, whole map display, localization inquiry and so on, and rendered the created special topic of the information of science and technology to farmer. It is advantageous to make analysis and decision to the distribution information of agricultural science and technology to farmer by GIS. The development steps with VB and the MapObjects are introduced in a lot of related books, such as MapObjects-GIS Programming (Xue Wei, 2004) and Getting Started with MapObjects (Mchael Zeiler, 1999). Fig. 6 shows the interface of the system.

\subsubsection{Database Operation}

There are all kinds of operations to database in Visual Basic, such as inquiry, adding, deletion, update, statistical classification and so on. The 


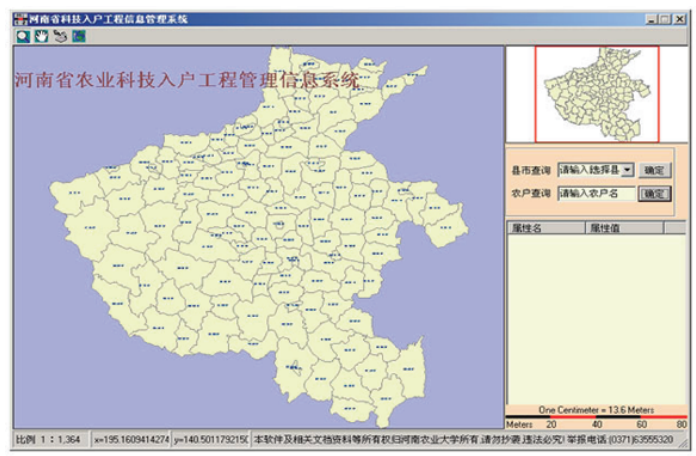

Fig. 6. The interface of the system

system took ADO component to realize operations to SQL Server 2000. Besides three components that are used to operate database: ADO component, DATA component, DAO component, user may also research and develop special components to operate database (Julia Case Bradley, 2003; Microsoft Corporation, 1999). Using SQL command, system realized inquiry and update to the information database of agricultural science and technology to farmer.

\section{CONCLUSION AND FUTURE WORKS}

System design has followed: scientific and solid system structure, practical and extensible function, artistic interface and so on (Huang Liuqing, 2005). The system has been applied to the information management of agricultural science and technology to farmer in HUA county, and facilitated information management, analysis and decision-making to the agricultural science and technology to farmer. The system may be popularized in all over the county, also the country.

Along with the continuous development of computer technology, Internet and WebGIS (Shang Wu, 2006; Wu Yun-chao, 2007; Liu Yi-jun, 2007), GIS will be applied and spread out deeply in the domain of "agriculture, country and farmer". In the following work, the system will take B/S and use network programming technology to realize the information management of agricultural science and technology to farmer based on WebGIS.

\section{ACKNOWLEDGEMENTS}

This study is supported by Henan Education Department tackling key problem of Science and technology Projects (Contract Number: 200510466005). Sincerely thanks are also due to the Hua County 


\section{Agricultural Technology Popularization Center for providing the data for} this study.

\section{REFERENCES}

Tang Wan-min, Jia Ming-qing, Wang Yan-chao. 2006. Promoting dominant technology popularization and application by the project of agricultural science and technology to farmer, Xian Dai Nong Ye Ke Ji, 5: 105-107 (in Chinese).

Yin Li-hui, XIA Sheng-ping. 2006. Effects of model project of agricultural sci \& tech, Hunan Agricultural Sciences, 2: 8-10 (in Chinese).

Http://www.gov.cn/jrzg/2006-10/17/content_415658.htm. 2006, 10. The project of agricultural science and technology to farmer put into practice by Ministry of Agriculture drives 4 million peasant households to increase production and income.

Alexander Köninger, Sigrid Bartel. 1998. 3d-Gis for Urban Purposes, GeoInformatica, 2(1): 79-103.

Yan Tai-lai, Zhu De-hai, Zhang Xiao-dong. 2005. To apply "3S" technology and carry on the concept of scientific development for agriculture, Journal of China Agricultural University, 10(6): 16-20 (in Chinese).

Wei Fu-quan, Xie Fang, Huang Tian-zhou, Sun Duan. 2004. Application of 3S technology in forestry, Forestry Prospect and Design, 1: 45-47 (in Chinese).

Li Li-wei, Xiao Ya-li, Liang Bao-song. 2006. Study on the GIS-based forest resources management information system, Henan Agricultural College Press, 5(40): 503-505 (in Chinese).

Gong Jian-ya. 2004. Review of the progress in contemporary GIS, Geomatics \& Spatial Information Technology, 27(1): 5-11 (in Chinese).

Wu Lun. 2001. Theory, method and application of GIS, Science publishing House, Beijing.

Gong Jian-ya, Du Dao-sheng, Li Qing-quan. 2004. Modern GIS, Science publishing House, Beijing.

Chen Zheng-jiang, Tang Guo-an, Ren Xiao-dong. 2005. Design and development of GIS, Science Press, Beijing.

Zheng Ke-feng, Zhu Li-li, Hu Wei-qun. 2005. System design and realization of agriculture geography information system, Zhejiang Agricultural Science, 4: 244-246 (in Chinese).

Yang Bao-zhu, Liu Feng, Li Xiang. 2005. Design and implement of crop management system based on WebGIS, Agricultural Network Information, 4: 18-25 (in Chinese).

Xue Wei. 2004. MapObjects-GIS Programming, Defense industry press, Beijing.

Mchael Zeiler. 1999. Getting started with MapObjects, (USA) ESRE Press.

Julia Case Bradley, Anita C. Millspaugh. 2003. Highlevel programming about Visual Basic 6.0, Tsinghua University Press, Beijing.

Microsoft Corporation (USA), 1999. The component reference manual of Microsoft Visual Basic 6.0, Beijing Hope Electron Press.

Huang Liu-qing. 2005. Becoming a component-oriented designer, Programmer Journal, 9: 133-135 (in Chinese).

Shang Wu. 2006. WebGIS:status and prospects, Geologcal Bulletin of China, 25(4): 533-537 (in Chinese).

Wu Yun-chao, Wang Wen, Niu Zheng, Song Guo-jun. 2007. Integrating ajax approach into WebGIS, Geography and Geo-Information Science, 23(2): 43-46 (in Chinese).

Liu Yi-jun, Hu Xiang-yun. 2007. Application of XML in WebGIS, Science Technology and Engineering, 7(6): 1095-1097, 1106 (in Chinese). 